lead to a spectrum of muscle diseases that range from congenital to adult-onset (1).

\section{Therapeutic implications}

Enzyme delivery is an effective means of treating some muscle diseases, but whether $\alpha \mathrm{DG}$ can be adequately targeted by enzyme replacement has not yet been addressed. The retroposon insertion responsible for FCMD induces missplicing of FKTN, and this could be corrected by antisense oligonucleotide treatment, which restored fukutin activity and normal $\alpha$ DG glycosylation (5). Therefore, combinatorial approaches to partially restore FKTN expression and augment activity through other enzymes may prove feasible for enhancing muscle function in the congenital muscular dystrophies. Furthermore, the work of Beedle suggests that the most effective targeting strategy might require treatment early in muscle development.

\section{Acknowledgments}

E.M. McNally is supported by NIH grants HL61322, AR052646, and NS072027 and by the Doris Duke Charitable Foundation.
Address correspondence to: Elizabeth M. McNally, Department of Medicine, Department of Human Genetics, The University of Chicago, 5841 S. Maryland, MC6088, Chicago, Illinois 60637, USA. Phone: 773.702.2672; Fax: 773.702.2681; E-mail: emcnally@uchicago.edu.

1. Muntoni F, Torelli S, Brockington M. Muscular dystrophies due to glycosylation defects. Neurotherapeutics. 2008;5(4):627-632.

2. Collins J, Bonnemann CG. Congenital muscular dystrophies: toward molecular therapeutic interventions. Curr Neurol Neurosci Rep. 2010;10(2):83-91.

3. Smalheiser NR, Schwartz NB. Cranin: a lamininbinding protein of cell membranes. Proc Natl Acad Sci U S A. 1987;84(18):6457-6461.

4. Kobayashi K, et al. An ancient retrotransposal insertion causes Fukuyama-type congenital muscular dystrophy. Nature. 1998;394(6691):388-392.

5. Taniguchi-Ikeda $M$, et al. Pathogenic exontrapping by SVA retrotransposon and rescue in Fukuyama muscular dystrophy. Nature. 2011; 478(7367):127-131.

6 . Takeda $S$, et al. Fukutin is required for maintenance of muscle integrity, cortical histiogenesis and normal eye development. Hum Mol Genet. 2003; 12(12):1449-1459.

7. Beedle AM, et al. Mouse fukutin deletion impairs dystroglycan processing and recapitulates muscular dystrophy. J Clin Invest. 2012;122(9):3330-3342.

8. Holtzer H, Jones KW, Yaffe D. Research group on neuromuscular diseases. A report on various aspects of myogenic cell culture with particular reference to studies on the muscular dystrophies. J Neurol Sci. 1975;26(1):115-124.

9. Chiba A, et al. Structures of sialylated O-linked oligosaccharides of bovine peripheral nerve alpha-dystroglycan. The role of a novel O-mannosyl-type oligosaccharide in the binding of alpha-dystroglycan with laminin. J Biol Chem. 1997;272(4):2156-2162.

10. Yoshida-Moriguchi T, et al. O-mannosyl phosphorylation of alpha-dystroglycan is required for laminin binding. Science. 2010;327(5961):88-92.

11. Inamori K, Yoshida-Moriguchi T, Hara Y, Anderson ME, Yu L, Campbell KP. Dystroglycan function requires xylosyl- and glucuronyltransferase activities of LARGE. Science. 2012;335(6064):93-96.

12. Grewal PK, Hewitt JE. Mutation of Large, which encodes a putative glycosyltransferase, in an animal model of muscular dystrophy. Biochim Biophys Acta. 2002;1573(3):216-224

13. Holzfeind PJ, et al. Skeletal, cardiac and tongue muscle pathology, defective retinal transmission, and neuronal migration defects in the Large(myd) mouse defines a natural model for glycosylationdeficient muscle - eye - brain disorders. Hum Mol Genet. 2002;11(21):2673-2687.

14. Willer $\mathrm{T}$, et al. ISPD loss-of-function mutations disrupt dystroglycan O-mannosylation and cause Walker-Warburg syndrome. Nat Genet. 2012; 44(5):575-580.

15. Nguyen HH, Jayasinha V, Xia B, Hoyte K, Martin PT. Overexpression of the cytotoxic T cell GalNAc transferase in skeletal muscle inhibits muscular dystrophy in mdx mice. Proc Natl Acad Sci U S A. 2002; 99(8):5616-5621.

\title{
FAM83A and FAM83B: candidate oncogenes and TKI resistance mediators
}

\author{
Steven Grant \\ Division of Hematology/Oncology, Virginia Commonwealth University Health Science Center, Richmond, Virginia, USA.
}

\begin{abstract}
The growth and survival of tumor cells can depend upon the expression of a single oncogene, and therapeutically targeting this oncogene addiction has already proven to be an effective approach in fighting cancer. However, it is also clear that cancer cells can adapt and become resistant to therapy through compensatory activation of downstream pathways that relieve the cell of its addicted phenotype. In this issue of the JCI, two groups - Lee et al. and Cipriano et al. - identify two related candidate oncogenes that might both contribute to therapeutic resistance to tyrosine kinase inhibitors (TKIs). If validated, this information could help to identify new targets for therapeutic interventions in breast cancer and possibly other cancers and may also assist in the development of strategies designed to overcome resistance to currently available TKIs.
\end{abstract}

The notions of oncogene addiction and resistance to targeted agents are inextricably intertwined. The success of agents

Conflict of interest: The author has declared that no conflict of interest exists.

Citation for this article: J Clin Invest. 2012; 122(9):3048-3051. doi:10.1172/JCI64412. that target aberrantly expressed bone fide oncogenic receptor tyrosine kinases (RTKs), such as BCR/ABL in the case of CML or EGFR in the case of diverse epithelial malignancies, rests on the presumption that transformed cells have become, in contrast to their normal counterparts, addicted to a particular pathway for their survival (1). Two of the pathways most frequently involved in this phenomenon are the RAS/RAF/MEK/ERK and the PTEN/PI3K/AKT/mTOR cascades, which are among the most commonly dysregulated pathways in cancer. More recently, attention has begun to focus on so-called "orthogonal" pathways and their contribution to the transformed phenotype (2). These pathways protect neoplastic cells from multiple forms of oncogenic stress (e.g., proteotoxic, oxidative, DNA damage-related, etc.) which accompany expression of oncogenes (e.g., $c-M y c)$ that confer survival or proliferation advantages on involved cells. However, an improved appreciation of the basis for the transformed phenotype also brings an understanding of the complex mechanisms capable of conferring resistance or sensitivity to targeted agents. For example, 


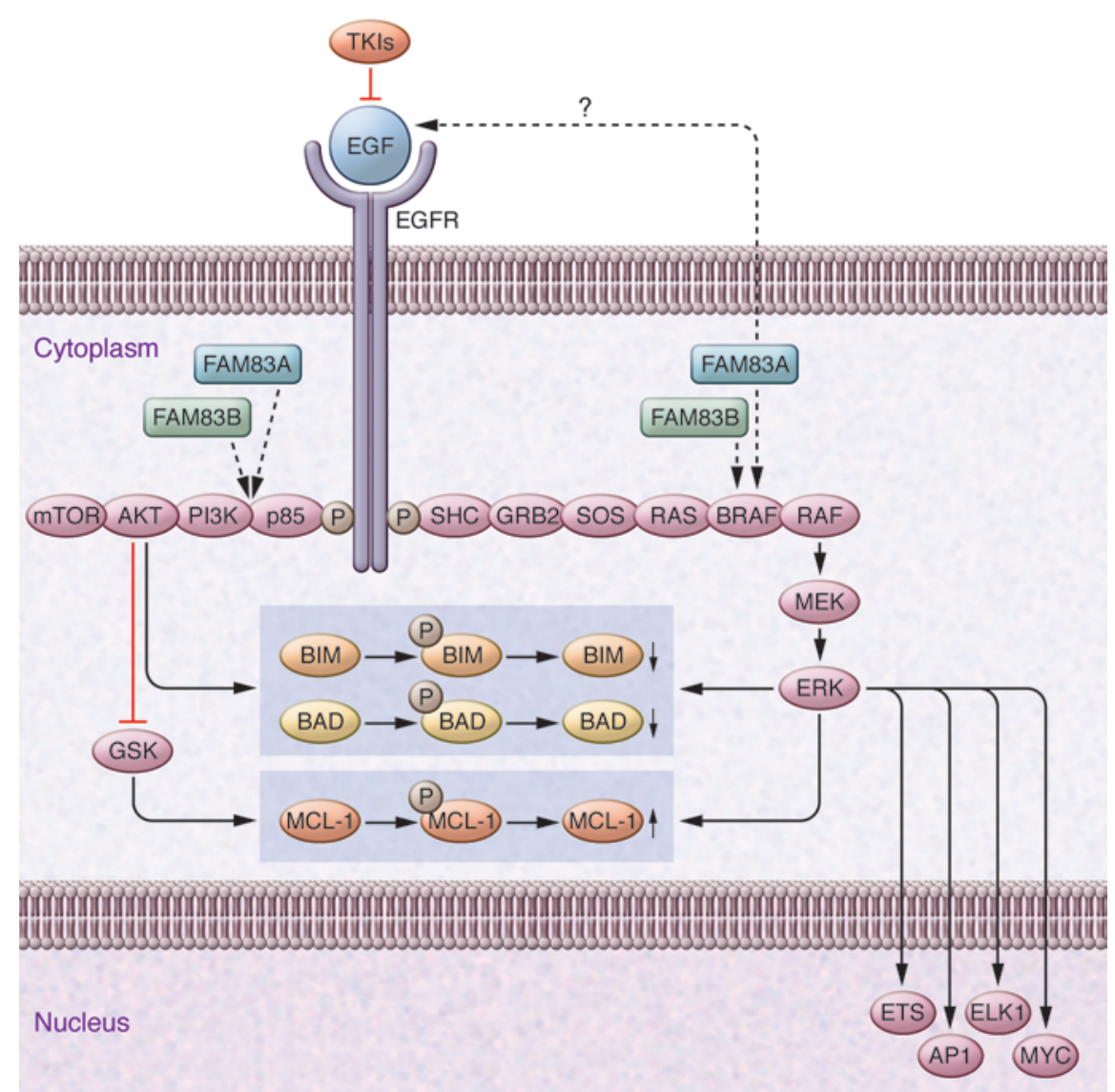

Figure 1

Hypothetical model by which candidate oncogenes such as FAM83A and FAM83B may influence transformed cell behavior, while simultaneously modifying responses to TKIs. Tyrosine kinases such as EGF are frequently dysregulated in cancer and signal downstream to multiple growth and survival pathways, including the RAS/RAF/MEK/ERK and the PI3K/AKT pathways. The prosurvival actions of these pathways are mediated, at least in part, through phosphorylation of proapoptotic and antiapoptotic BCL-2 family proteins (e.g., BIM, BAD, and MCL-1), leading to alterations in the relative abundance of as well as interactions between these proteins. Note that AKT acts indirectly to spare MCL-1 by inhibiting glycogen synthase kinase-mediated (GSK-mediated) MCL-1 phosphorylation and degradation. TKIs act by blocking EGF signaling to these key pathways, and modification of the balance between BCL-2 family proteins may contribute to TKI-mediated lethality. As shown by the dashed arrows, FAM83A and FAM83B could theoretically act downstream of EGF but upstream of BRAF and p85-PI3K to promote activation of these survival pathways, leading to attenuation of cell death. FAM83A and FAM83B may also, through a yet to be defined mechanism, modify the extent or tonicity of EGF signaling itself. By acting at sites downstream of EGF, FAM83A and FAM83B may thereby bypass the block to EFG survival signaling induced by TKIs, effectively relieving the cell of its addiction to this survival pathway. Such a phenomenon could theoretically be prevented by either developing inhibitors of FAM83A and/or FAM83B that interfere with their interactions with proteins such as BRAF or specifically targeting downstream components of the pathways responsible for the prosurvival actions of these proteins. Adapted with permission from Nature Reviews Cancer (17).

resistance of cells to tyrosine kinase inhibitors (TKIs) may reflect not only mutations that prevent drug binding (3), but also cross-talk between and compensatory activation of downstream pathways that relieve the cell of its addicted phenotype. In the case of EGFR inhibitors, the latter phenomenon often involves perturbations in the PI3K/AKT/mTOR or RAS/RAF/ MEK/ERK pathways (4). Adding further to the complexity are the observations that mutations in pathway components (e.g., activating EGFR mutation in lung cancer, ref. 5, and B-RAF mutation in melanoma) may render those tumors exquisitely sensitive to inhibitors of those kinases (6).
Together, these considerations have concrete implications for the characterization of candidate oncogenes as well as attempts to understand their therapeutic effects. For instance, it goes without saying that any such candidates would be required to influence the biologic behavior of the cells that express them, for example, by altering the proliferative potential or growth factor dependence. However, the question now is whether and how such putative oncogenes might modify the responses of expressing cells to currently available RTK inhibitors.

\section{Oncogenic potential}

Several of these themes are highlighted by articles appearing in the current issue of the JCI, in which two groups, using related approaches, focused on members of the family with sequence similarity, members $A$ and B (FAM83A and FAM83B) $(7,8)$. Although differences between these reports clearly exist, the findings and conclusions of these groups exhibit marked similarities and suggest that these genes may contribute both to the pathophysiology of breast cancer as well as to resistance to EGFR inhibitors. In the study by Lee et al., from the laboratory of Mina J. Bissell, the authors used a threedimensional phenotypic reversion assay in breast cancer cells to identify FAM83A as a candidate oncogene capable of conferring EGFR inhibitor resistance (7). This group observed that forced FAM83A expression enhanced breast cancer cell proliferation and invasiveness, while at the same time increasing resistance to TKIs. Conversely, depletion of FAM83A reverted the malignant phenotype, reduced tumor growth in immunocompromised mice, and increased TKI responsiveness. Interestingly, expression of FAM83A was associated with increased phosphorylation of members of the RAS/ $\mathrm{RAF} / \mathrm{MEK} / \mathrm{ERK}$ and PI3K/AKT/mTOR pathways. These findings, along with evidence that FAM83A expression correlates inversely with prognosis of patients with breast cancer, raise the possibility that this gene may contribute both to breast cancer aggressiveness as well as TKI resistance. Significantly, FAM83A was observed to interact with c-RAF and PI3K p85 (7), components of EGFR downstream survival pathways, which, as noted previously, have both been implicated in EGFR inhibitor resistance.

In the study by Cipriano et al., from the laboratory of Mark W. Jackson, the authors used a validation-based insertional mutagenesis screen and identified a related gene, FAM83B, capable of transforming human 
mammary epithelial cells (8). As observed in the case of FAM83A, silencing FAM83B diminished the growth of these cells, while forced expression reduced their sensitivity to TKIs. In addition, the consequences of FAM83B expression were related to interactions with CRAF, accompanied by activation of ERK1/2 as well as AKT, pathways previously implicated in circumvention of TKI-mediated lethality. Finally, FAM83B expression resulted in more sustained EGFR phosphorylation (8), a phenomenon that might plausibly contribute to TKI resistance. Together, these companion studies identify two related candidate oncogenes that might both contribute to EGFR resistance, possibly through physical or other interactions with CRAF and potentially other common downstream cytoprotective pathways. If validated, this information could help to identify new targets for therapeutic interventions in breast cancer and possibly other cancers and may also assist in the development of strategies designed to overcome resistance to currently available TKIs.

\section{Drivers or passengers?}

Although the results of and conclusions drawn from these studies appear logical and consistent, a number of questions remain to be addressed. For one thing, while dysregulation of FAM83A and FAM83B appears to occur relatively frequently in human tumor specimens, it is uncertain whether they are primarily responsible for transformation in this setting or instead represent passenger mutations that play more of a permissive role. The increased expression of these genes in diverse tumor types complicates the task of defining their precise etiological role in tumorigenesis. Consequently, considerable work will be required to define the role of FAM83A and FAM83B in carcinogenesis more definitely.

One of the more interesting features of the two studies is that they focus attention on the interdependent phenomena of oncogene addiction and drug resistance. More specifically, both studies shine a spotlight on the role of RTK downstream pathways, particularly the RAS/RAF/MEK/ERK and $\mathrm{PI} 3 \mathrm{~K} / \mathrm{AKT}$ cascades, in both the transforming activities of FAM83A and FAM83B as well as escape from the lethal effects of TKIs. It has long been recognized that aberrant EGFR signaling exerts its prosurvival effects, at least in part, through activation of these pathways (9). Moreover, resistance to TKIs has been related to constitutive or stimulated activation of AKT or MEK/ERK (10, 11). Thus, FAM83A and FAM83B may function by bypassing RTK dependence through activation of ERK1/2, AKT, and possibly other kinases, effectively relieving the cell of its addiction to EGFR. On the other hand, the observation that FAM83B may prolong EGFR signaling raises the possibility that this gene may act at a more proximal point in the network to reduce TKI lethality. To answer these questions definitively, a more systematic functional analysis of FAM83Aand FAM83B-mediated perturbations in these signaling networks in the presence of TKIs will be required.

Another issue to be addressed is identification of the mechanism(s) by which FAM83A- and FAM83B-induced activation of MAPK and other signaling pathways promotes transformed cell survival, particularly in the presence of TKIs. It is generally thought that the prosurvival effects of MAPK and AKT are integrated at the level of BCL-2 family proteins, including BIM and BAD (4). In this context, it has been shown that TKI responsiveness can be highly dependent upon expression of the proapoptotic BH3-only protein BIM, and a very recent study identified a common BIM deletion polymorphism as a mediator of intrinsic resistance to TKIs (12). Significantly, AKT- and ERK-mediated BIM phosphorylation leads to its ubiquitination and proteasomal degradation $(13,14)$. It would therefore be very interesting to determine what effect, if any, expression of FAM83A or FAM83B might have on BIM abundance, and whether such actions might contribute to TKI resistance. In addition, it is recognized that, aside from the total and relative levels of proapoptotic to antiapoptotic BCL-2 family members, cell survival may also be determined by physical interactions between these proteins. In this context, ERK phosphorylation of BIM diminishes its proapoptotic association with BAX (15). Consequently, FAM83Aand FAM83B-mediated activation of the CRAF-MAPK axis may diminish TKI responsiveness by altering Bim interactions with antiapoptotic proteins such as MCL-1 (16). A summary of the hypothetical basis by which FAM83A and FAM83B might influence the response of tumor cells to TKIs is illustrated in Figure 1.

\section{The starting point for translation}

Finally, the studies by Lee et al. and Cipriano et al. raise the possibility that FAM83A and FAM83B, or the proteins that they encode, could represent candidate targets suitable for therapeutic intervention. Clearly, the ultimate success of such an approach will depend upon better understanding of the functions of these proteins, which, based upon preliminary findings, appear to be quite pleiotropic in nature. In this regard, evidence is presented suggesting that the activation of CRAF, and resulting transformation, involves FAM83B binding (8). While such protein-protein interactions are no longer considered "undruggable," successful development of such inhibitors will hardly be a trivial enterprise. Even if this approach is not feasible, an increased understanding of the downstream targets responsible for FAM83Aor FAM83B-mediated TKI resistance will highlight complementary therapeutic targets that might specifically enhance TKI activity in patients with tumors exhibiting dysregulated FAM83A or FAM83B. As labor intensive as this approach may be, the current limitations of TKIs in the treatment of breast cancer and other tumor types justifies such efforts.

\section{Acknowledgments}

This work was supported by grants from the NIH (CA93738, CA 100866-01, CA130805, and CA142509) and by the Multiple Myeloma Research Foundation and the Leukemia and Lymphoma Society of America.

Address correspondence to: Steven Grant, Division of Hematology/Oncology, Virginia Commonwealth University Health Science Center, Room 234 Goodwin Research Building, 401 College Street, Richmond, Virginia 23229, USA. Phone: 804.828.5211; Fax: 804.828.5164; E-mail: stgrant@vcu.edu.

1. Weinstein IB, Joe AK. Mechanisms of disease: Oncogene addiction-a rationale for molecular targeting in cancer therapy. Nat Clin Pract Oncol. 2006;3(8):448-457.

2. Luo J, Solimini NL, Elledge SJ. Principles of cancer therapy: oncogene and non-oncogene addiction. Cell. 2009;136(5):823-837.

3. Shah NP, et al. Multiple BCR-ABL kinase domain mutations confer polyclonal resistance to the tyrosine kinase inhibitor imatinib (STI571) in chronic phase and blast crisis chronic myeloid leukemia. Cancer Cell. 2002;2(2):117-125.

4. She QB, Solit DB, Ye Q, O'Reilly KE, Lobo J, Rosen $\mathrm{N}$. The BAD protein integrates survival signaling by EGFR/MAPK and PI3K/Akt kinase pathways in PTEN-deficient tumor cells. Cancer Cell. 2005;8(4):287-297.

5. Lynch TJ, et al. Activating mutations in the epidermal growth factor receptor underlying responsiveness of non-small-cell lung cancer to gefitinib. NEngl J Med. 2004;350(21):2129-2139. 
6. Chapman PB, et al. Improved survival with vemurafenib in melanoma with BRAF V600E mutation. NEngl J Med. 2011;364(26):2507-2516.

7. Lee S-Y, et al. FAM83A confers EGFR-TKI resistance in breast cancer cells and in mice. J Clin Invest. 2012;122(9):3211-3220.

8. Cipriano R, et al. FAM83B mediates EGFR- and RAS-driven oncogenic transformation. J Clin Invest. 2012;122(9):3197-3210.

9. Avraham R, Yarden Y. Feedback regulation of EGFR signalling: decision making by early and delayed loops. Nat Rev Mol Cell Biol. 2011;12(2):104-117.

10. She QB, Solit D, Basso A, Moasser MM. Resistance to gefitinib in PTEN-null HER-overexpressing tumor cells can be overcome through restoration of PTEN function or pharmacologic modulation of constitu- tive phosphatidylinositol 3'-kinase/Akt pathway signaling. Clin Cancer Res. 2003;9(12):4340-4346.

11. Normanno N, et al. The MEK/MAPK pathway is involved in the resistance of breast cancer cells to the EGFR tyrosine kinase inhibitor gefitinib. Cell Physiol. 2006;207(2):420-427.

12. Ng KP, et al. A common BIM deletion polymorphism mediates intrinsic resistance and inferior responses to tyrosine kinase inhibitors in cancer. Nat Med. 2012;18(4):521-528.

13. Qi XJ, Wildey GM, Howe PH. Evidence that Ser87 of BimEL is phosphorylated by Akt and regulates BimEL apoptotic function. J Biol Chem. 2006;281(2):813-823.

14. Ley R, Ewings KE, Hadfield K, Howes E, Balmanno $\mathrm{K}$, Cook SJ. Extracellular signal-regulated kinases
$1 / 2$ are serum-stimulated " $\mathrm{Bim}(\mathrm{EL})$ kinases" that bind to the BH3-only protein Bim(EL) causing its phosphorylation and turnover. $J$ Biol Chem. 2004;279(10):8837-8847.

15. Harada H, Quearry B, Ruiz-Vela A, Korsmeyer SJ. Survival factor-induced extracellular signal-regulated kinase phosphorylates BIM, inhibiting its association with BAX and proapoptotic activity. Proc Natl Acad Sci U S A. 2004;101(43):15313-15317.

16. Ewings KE, et al. ERK1/2-dependent phosphorylation of $\operatorname{Bim}(\mathrm{EL})$ promotes its rapid dissociation from Mcl-1 and $\mathrm{Bcl}-\mathrm{x}(\mathrm{L}) . E M B O J .2007$; 26(12):2856-2867.

17. Kolch W, Pitt A. Functional proteomics to dissect tyrosine kinase signalling pathways in cancer. Nat Rev Cancer. 2010;10(9):618-629.

\title{
Mining the secrets of the CSF: developing biomarkers of neurodegeneration
}

\author{
William Z. Potter
}

National Institute of Mental Health, Bethesda, Maryland, USA.

\begin{abstract}
Our ability to track the progression of neurological disorders like Parkinson's disease (PD) is hampered by a lack of biomarkers, rendering the neuronal changes that underlie clinical symptoms largely a mystery. In this issue of the JCI, Fanara et al. report the development of an innovative approach to biomarker development. They describe a method to measure axonal microtubule function via cerebrospinal fluid (CSF) sampling and use this technique to provide evidence of deficiencies in this process in PD patients. This both sheds light on the pathophysiology of PD and has implications for the more general problem of developing biomarkers for any brain process.
\end{abstract}

\section{The problem}

The underlying pathologic processes driving the symptoms of neurodegenerative disease cannot be followed in living subjects without better biomarkers. The difficulty in identifying such biomarkers of disease progression has impeded the development of therapeutic strategies to combat it. The study by Fanara et al. on cerebrospinal fluid (CSF) kinetic biomarkers of axonal transport in this issue of the $J C I$ marks an important contribution to the field of measuring difficult-to-assess brain processes in vivo (1). The authors' work expands on a series of earlier studies in murine models that used heavy water $\left({ }^{2} \mathrm{H}_{2} \mathrm{O}\right)$ labeling to investigate microtubule dynamics and stabilization by taxanes with neuroprotective potential $(2,3)$. Here,

Conflict of interest: William Z. Potter holds stock in Lilly and Merck and has served as a consultant for Envoy.

Citation for this article: J Clin Invest. 2012; 122(9):3051-3053. doi:10.1172/JCI65309.
Fanara et al. systematically developed a biomarker of microtubule function (1), and their work has implications for the more general problem of developing biomarkers for any brain process.

Ideally, in the development of biomarkers intended for human application, one needs a model system in which variables can be controlled and manipulated. Although genetic or lesion models of human disease are available, to be clinically relevant, the direct measures possible in animal brain coupled to a biomarker analysis must be translatable to humans. CSF is an attractive sample source. Although technical issues involved in sampling from mice have discouraged many groups, Fanara et al. provide a compelling case that it is possible to relate a pathologic process in a murine model to a specific CSF analyte measurable in mice and humans.

\section{CSF as a biomarker source}

To appreciate the potential importance of a wider application of this strategy of bio- marker development, we must consider what traditional human CSF studies have and have not been able to deliver in the past. Human CSF has been a source of biomarkers, especially as an index of drug action, for decades. For instance, antidepressants were shown to produce hypothesized effects on CSF amine neurotransmitter metabolites in the early 1970 s (4). The kinetic principles for interpreting concentrations of analytes in CSF were established during this same period, including the use of stable isotopes (5). Recently, especially in Alzheimer's disease (AD), discovery proteomic assays have identified a number of analytes beyond amyloid- $\beta$ and phospho-tau, increasing interest in CSF as a source of biomarkers of disease (6). Even when robust relationships can be established between a CSF analyte and a disease process or drug action, gaps remain in our knowledge regarding how best to interpret a concentration change. Definitive interpretations require more precise knowledge of the sites of formation of the analyte as well as the processes that control its concentration in CSF.

Figure 1 provides a minimal model of the processes that would need to be quantitatively understood in order to fully interpret behavior of any CSF analyte. Even with simplifying assumptions, such as common rate constants for molecule formation and movement from all brain tissues into the CSF, other rate constants are relevant to our understanding; these include not only direct egress into peripheral compart- 